\title{
Decay of charge deposited on the wall of gaseous void
}

\author{
McAllister, lain Wilson
}

Published in:

IEEE Transactions on Electrical Insulation

Link to article, DOI:

$10.1109 / 14.204872$

Publication date:

1992

Document Version

Publisher's PDF, also known as Version of record

Link back to DTU Orbit

Citation (APA):

McAllister, I. W. (1992). Decay of charge deposited on the wall of gaseous void. IEEE Transactions on Electrical Insulation, 27(6), 1202-1207. https://doi.org/10.1109/14.204872

\section{General rights}

Copyright and moral rights for the publications made accessible in the public portal are retained by the authors and/or other copyright owners and it is a condition of accessing publications that users recognise and abide by the legal requirements associated with these rights.

- Users may download and print one copy of any publication from the public portal for the purpose of private study or research.

- You may not further distribute the material or use it for any profit-making activity or commercial gain

- You may freely distribute the URL identifying the publication in the public portal

If you believe that this document breaches copyright please contact us providing details, and we will remove access to the work immediately and investigate your claim 


\title{
COMMUNICATION
}

\section{Decay of Charge Deposited on the Wall of a Gaseous Void}

\author{
I. W. McAllister \\ Electric Power Engineering Department \\ Technical University of Denmark, Lyngby, Denmark
}

\begin{abstract}
Following partial discharge activity within a gaseous void, charges will accumulate on the wall of the void. In the present paper, the decay of such charges due to surface currents at the void wall is studied analytically, and the factors affecting this decay are indicated.
\end{abstract}

\section{INTRODUCTION}

$\mathrm{P}$ ARTIal discharges can occur in a gaseous void within solid insulation. Such discharges deposit free charges on the void wall. Depending on the polarity of the applied field, the electric field produced by these wall charges can either inhibit or promote further discharge development. However, should the void wall exhibit a finite surface conductivity, the wall charges will become neutralized in time by surface currents at the wall. This behavior will lead to a time-dependent wall charge field. In the present paper, the decay of the surface charge is investigated theoretically, and the factors influencing this decay are discussed.

\section{CONTINUITY EQUATION FOR SURFACE CURRENTS}

HE surface in question is considered to be the inter-
face between a medium 'a' and a medium ' $b$ '. The
latter is a sphere of radius $R$, while the former occupies
the remaining space which extends to infinity. Hence with
respect to spherical coordinates $r, \theta, \psi$ the interface is
represented by the surface $r=R$. As we are examining
the behavior of surface currents due to an interface which exhibits a finite surface conductivity $\Gamma$, the relevant continuity equation is

$$
\vec{\nabla}_{s} \cdot \vec{K}+\frac{\partial \sigma}{\partial t}=0
$$

where $\vec{K}$ is the surface current density, $\sigma$ the surface charge density and $t$ time. $\vec{\nabla}_{s} \cdot \vec{K}$ represents the surface divergence of $\vec{K}[1] . \quad \vec{K}$ is related to the tangential electric field strength $\vec{E}_{t}$ at the surface by

$$
\vec{K}=\Gamma \vec{E}_{t}
$$

Employing the continuity equation in the above form implies that, in this study, the volume conduction current density $\vec{J}$ is zero in each medium. Before proceeding with the present analysis, it will be assumed that the surface conductivity is homogeneous and isotropic such that a linear relationship exists between $\vec{K}$ and $\vec{E}_{t}$.

For the present class of boundary value problems, the general potential functions associated with the two media bounding the interface can be expressed as [2]

$$
\begin{aligned}
& \Phi_{a}(r, \theta, \psi, t)=\varphi_{a}(r, \theta, \psi) T(t) \\
& \Phi_{b}(r, \theta, \psi, t)=\varphi_{b}(r, \theta, \psi) T(t)
\end{aligned}
$$

where $\varphi_{a}$ and $\varphi_{b}$ represent the time zero potential distributions in medium ' $a$ ' and medium ' $b$ ', respectively, and 
are thus solutions of Laplace's equation. The temporal variation $T$ is a function of $t$ alone with $T(0)=1$.

By considering the field boundary conditions associated with $\vec{K}$ and $\sigma$, it is possible to derive the equivalent continuity equation to be fulfilled by the potential functions $\Phi_{a}$ and $\Phi_{b}$ at the spherical interface [2]. For $r=R$, one obtains

$$
\frac{\Gamma}{r^{2}} \frac{\partial}{\partial r}\left[r^{2} \frac{\partial \varphi}{\partial r}\right] T+\left[\varepsilon_{b} \frac{\partial \varphi_{b}}{\partial r}-\varepsilon_{a} \frac{\partial \varphi_{a}}{\partial r}\right] \frac{d T}{d t}=0
$$

In (5) the form of the first term, which is related to $\vec{\nabla}_{8}$. $\vec{K}$, arises because the potential distributions in the two media bounding the interface are solutions of Laplace's equation. Hence as this term is concerned with the second derivative of the potential, the term can be derived from either $\varphi_{a}$ or $\varphi_{b}$, i.e. the lack of a potential subscript in this term is not a misprint. In deriving (5), the orientation of the interface is such that $r_{a} \geqslant r_{b}$. In addition, it must be emphasized that the derivation of (5) is based on the assumption of a constant $\Gamma$.

As the bracketed terms in (5) are independent of $t$, the solution to this differential equation is

$$
T=\exp (-\lambda t)
$$

with

$$
\lambda=\frac{\frac{\Gamma}{r^{2}} \frac{\partial}{\partial r}\left[r^{2} \frac{\partial \varphi}{\partial r}\right]}{\left[\varepsilon_{b} \frac{\partial \varphi_{k}}{\partial r}-\varepsilon_{a} \frac{\partial \varphi_{a}}{\partial r}\right]}
$$

Consequently the general potential solutions are

$$
\begin{aligned}
& \Phi_{a}=\varphi_{a} \exp (-\lambda t) \\
& \Phi_{b}=\varphi_{b} \exp (-\lambda t)
\end{aligned}
$$

$\varphi_{a}$ and $\varphi_{b}$ are deduced from the interfacial charge distribution at time $t=0$.

\section{APPLICATION TO A SPHERICAL VOID}

$$
\begin{aligned}
& \text { s the initial discharge development in the void is pre- } \\
& \text { sumed to be axially symmetric, we consider the wall } \\
& \text { charge distribution to exhibit the same symmetry. In } \\
& \text { such situations, the appropriate solutions for the time ze- } \\
& \text { ro potentials are } \\
& \qquad \varphi_{a}=\sum_{n=0}^{\infty} A_{n}\left[\frac{R}{r}\right]^{n+1} P_{n}(\cos \theta) \text { for } r \geqslant R \quad \text { (10) } \\
& \qquad \varphi_{b}=\sum_{n=0}^{\infty} A_{n}\left[\frac{r}{R}\right]^{n} P_{n}(\cos \theta) \text { for } r \leqslant R
\end{aligned}
$$

where $P_{n}(\cos \theta)$ is a Legendre polynomial. At the void wall $(r=R), \varphi_{a}$ and $\varphi_{b}$ automatically fulfill the potentialequality requirement. As the solutions for $\varphi_{a}$ and $\varphi_{b}$ in terms of spherical coordinates consist of infinite series, (7) cannot be used directly to determine the relevant $\lambda$. In this situation reference must be made to (5) from which, upon differentiating (10) and (11), we find that, for $r=R$,

$$
\lambda_{n}=\frac{n(n+1)}{(n+1) \varepsilon_{r a}+n \varepsilon_{r b}}\left[\frac{1}{\tau}\right]
$$

with $\tau=\varepsilon_{o} R / \Gamma$. In (12), $\varepsilon_{r}$ is used to denote relative permittivity. The potential solutions associated with the two media are thus

$$
\begin{gathered}
\Phi_{a}=\sum_{n=0}^{\infty} A_{n}\left[\frac{R}{r}\right]^{n+1} P_{n}(\cos \theta) \exp \left[\frac{-n(n+1)}{(n+1) \varepsilon_{r a}+n \varepsilon_{r b}} \frac{t}{\tau}\right] \\
\Phi_{b}=\sum_{n=0}^{\infty} A_{n}\left[\frac{r}{R}\right]^{n} P_{n}(\cos \theta) \exp \left[\frac{-n(n+1)}{(n+1) \varepsilon_{r a}+n \varepsilon_{r b}} \frac{t}{\tau}\right]
\end{gathered}
$$

To determine $A_{n}$ it is necessary to consider the nature of the initial charge distribution.

As a first approach, let $q$ be a point charge located on the void wall coincident with the axis of symmetry ( $r=$ $R, \theta=0$ ). From the potential solution for a dielectric sphere and a point charge given in standard textbooks $[3,4]$, we can deduce that

$$
A_{n}=\frac{q}{4 \pi \varepsilon_{o} R}\left[\frac{2 n+1}{(n+1) \varepsilon_{r a}+n \varepsilon_{r b}}\right]
$$

and thus the associated potential solutions are

$$
\begin{gathered}
\Phi_{a}=\frac{q}{4 \pi \varepsilon_{o} R} \sum_{n=0}^{\infty}\left\{\frac{2 n+1}{(n+1) \varepsilon_{r a}+n \varepsilon_{r b}}\left[\frac{R}{r}\right]^{n+1} \times\right. \\
\left.P_{n}(\cos \theta) \exp \left[\frac{-n(n+1)}{(n+1) \varepsilon_{r a}+n \varepsilon_{r b}} \frac{t}{\tau}\right]\right\} \\
\Phi_{b}=\frac{q}{4 \pi \varepsilon_{o} R} \sum_{n=0}^{\infty}\left\{\frac{2 n+1}{(n+1) \varepsilon_{r a}+n \varepsilon_{r b}}\left[\frac{r}{R}\right]^{n} \times\right. \\
\left.P_{n}(\cos \theta) \exp \left[\frac{-n(n+1)}{(n+1) \varepsilon_{r a}+n \varepsilon_{r b}} \frac{t}{\tau}\right]\right\}
\end{gathered}
$$

For $\varepsilon_{r a}=1$ and $\varepsilon_{r b}>1,(16)$ and (17) represent the potential solutions for a charged, insulating spherical particle. As $t \rightarrow \infty$, the charge will become dispersed around the spherical surface to form a shell of uniform charge density. Confirmation of this behavior is obtained on expanding the series in (16) and (17) and taking the limit for $t \rightarrow \infty$.

A void is however a closed volume, and thus the net charge within any void following partial discharge activity will remain zero. Consequently to represent this condition, it is necessary to consider a second wall charge, of 
opposite polarity, located diametrically opposite the first charge, i.e. $-q$ at $r=R, \theta=\pi$. Using the principle of superposition, we can obtain the composite solutions for $\Phi_{a}$ and $\Phi_{b}$ by simply adding appropriate solutions of the types given in (16) and (17). For $-q, P_{n}(\cos \theta)$ is replaced by $P_{n}(\cos [\pi-\theta])$. Because we have

$$
P_{n}(\cos \theta)-P_{n}(-\cos \theta)=0
$$

for $n$ even, while for $n$ odd

$$
P_{n}(\cos \theta)-P_{n}(-\cos \theta)=2 P_{n}(\cos \theta)
$$

we can express the composite potentials in a more convenient form on replacing $n$ by $2 n+1$. This substitution leads to

$$
\begin{aligned}
\Phi_{a 0}= & \frac{q}{2 \pi \varepsilon_{o} R} \sum_{n=0}^{\infty}\left\{\frac{4 n+3}{(2 n+2) \varepsilon_{r a}+(2 n+1) \varepsilon_{r b}} \times\right. \\
& {\left[\frac{R}{r}\right]^{2 n+2} P_{2 n+1}(\cos \theta) \times } \\
& \left.\exp \left[\frac{-(2 n+1)(2 n+2)}{(2 n+2) \varepsilon_{r a}+(2 n+1) \varepsilon_{r b}} \frac{t}{\tau}\right]\right\} \\
\Phi_{b 0}= & \frac{q}{2 \pi \varepsilon_{o} R} \sum_{n=0}^{\infty}\left\{\frac{4 n+3}{(2 n+2) \varepsilon_{r a}+(2 n+1) \varepsilon_{r b}} \times\right. \\
& {\left[\frac{r}{R}\right]^{2 n+1} P_{2 n+1}(\cos \theta) \times } \\
& \left.\exp \left[\frac{-(2 n+1)(2 n+2)}{(2 n+2) \varepsilon_{r a}+(2 n+1) \varepsilon_{r b}} \frac{t}{\tau}\right]\right\}
\end{aligned}
$$

The subscript 0 indicates that these potential functions relate to a zero net charge condition in the void and hence, as $t \rightarrow \infty$, the values of both $\Phi_{a 0}$ and $\Phi_{b 0}$ should tend to zero. This condition is fulfilled, because $n=0$ in (20) and (21) does not produce a time-independent term. With $\varepsilon_{r b}=1$, the above expressions will represent the potential variations in the bulk material $\left(\Phi_{a 0}\right)$ and in the void $\left(\Phi_{b 0}\right)$ when a simple point-charge configuration is used to simulate the void wall charges at time zero.

\section{SURFACE CHARGE DENSITY AT VOID WALL}

$\mathrm{Z}^{\mathrm{or}}$ the situation under discussion, the surface charge $\mathrm{H}$ density $\sigma$ at the void wall is given by

$$
\sigma=\varepsilon_{b} \frac{\partial \Phi_{b}}{\partial r}-\varepsilon_{a} \frac{\partial \Phi_{a}}{\partial r} \quad \text { for } r=R
$$

Hence upon differentiating (20) and (21) and inserting in (22), we obtain for $\varepsilon_{r b}=1$

$$
\begin{aligned}
\sigma=2 \sigma_{o} & \sum_{n=0}^{\infty}\left\{(4 n+3) P_{2 n+1}(\cos \theta) \times\right. \\
& \left.\exp \left[\frac{-(2 n+1)(2 n+2)}{(2 n+2) \varepsilon_{r a}+(2 n+1)} \frac{t}{\tau}\right]\right\}
\end{aligned}
$$

where

$$
\sigma_{o}=\frac{q}{4 \pi R^{2}}
$$

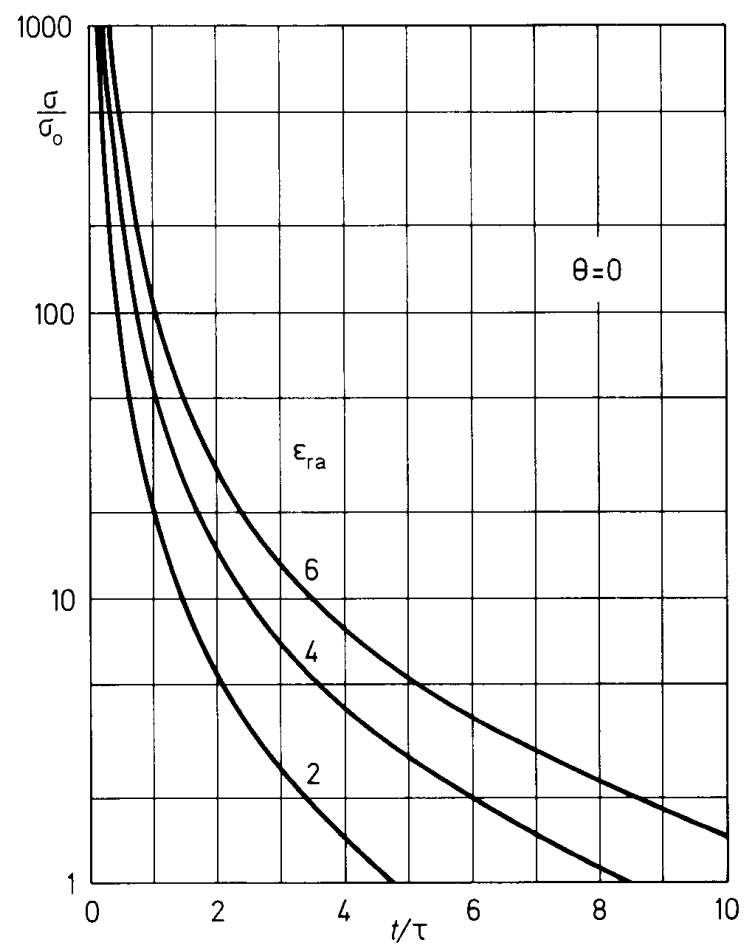

Figure 1.

Temporal variation of the surface charge density $\sigma$ at the time-zero charge location.

The temporal variation of $\sigma$ at the time-zero location of the charge is illustrated in Figure 1. $\sigma$ decays monotonically with $t$, but as the value of $\sigma$ is obtained from the summation of many exponential terms, see (23), the decay is not truly exponential in nature. Moreover, the greater the value of the bulk permittivity, the slower will be the observed the rate of this decay.

At locations which are charge-free at $t=0, \sigma$ increases initially to some maximum value and thereafter decays, see Figure 2. The value of the bulk permittivity influences both the time to the maximum $\sigma$-value $\left(t_{m a x}\right)$ and the value itself $\left(\sigma_{\max }\right)$. The variation in $\sigma_{\max }$ is relatively minor. However, both $\sigma_{\max }$ and $t_{\max }$ are strongly influenced by the proximity of the surface location in question to that of the time-zero source position.

The variation of $\sigma$ around the void wall is shown in Figures 3 and 4 . The former indicates the influence of $\varepsilon_{\text {ra }}$ for $t$ constant, whereas the latter shows the evolution 


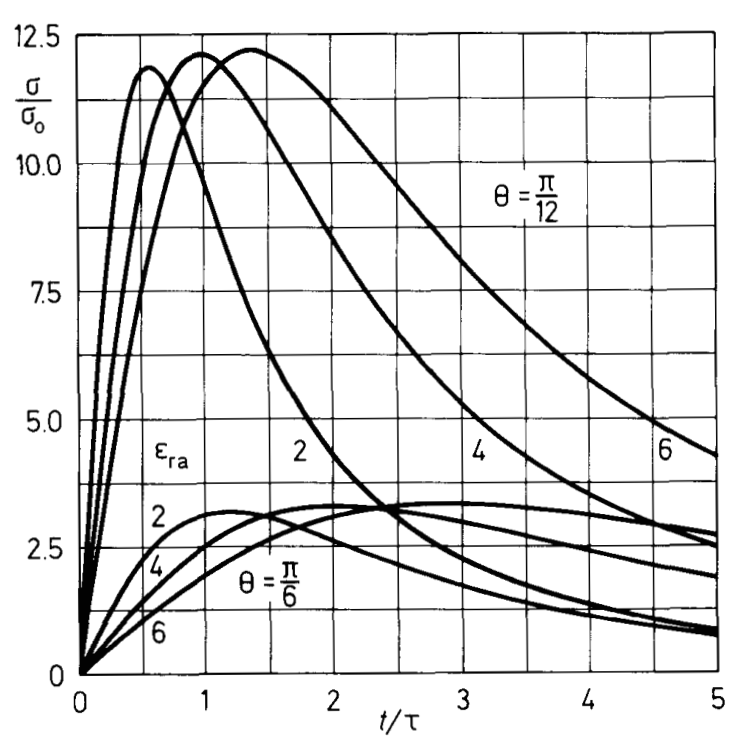

Figure 2.

Temporal variation of the surface charge density $\sigma$ at locations which are charge-free at time zero.

of $\sigma$ for $\varepsilon_{r a}=4$. It is evident from Figures 3 and 4 that wall charge takes a considerable number of time constants $(>5 \tau)$ to disperse, and that the absolute time is strongly influenced by the bulk permittivity.

\section{AXIAL ELECTRIC FIELD ACROSS THE VOID}

THE repetition rate of void discharges is controlled by I the time taken to re-establish a void field of sufficient magnitude to support discharge growth. Among other parameters, this time will be influenced by the decay of the field produced by wall charges. As a means of portraying this field, the variation of the field strength $E_{a x}$ along the void axis is considered. With reference to $E_{a x}$ we have $\left(\partial \Phi_{b} / \Phi \theta\right)=0$ and thus the axial field strength is given simply by

$$
E_{a x}=-\frac{\partial \Phi_{b}}{\partial r}
$$

Hence upon differentiating (21) we obtain, for $\varepsilon_{r b}=1$,

$$
\begin{aligned}
E_{a x}=- & 2 E_{o} \sum_{n=0}^{\infty}\left\{\frac{(4 n+3)(2 n+1)}{(2 n+2) \varepsilon_{r a}+(2 n+1)}\left[\frac{r}{R}\right]^{2 n} \times\right. \\
& \left.P_{2 n+1}(\cos \theta) \exp \left[\frac{-(2 n+1)(2 n+2)}{(2 n+2) \varepsilon_{r a}+(2 n+1)} \frac{t}{\tau}\right]\right\}
\end{aligned}
$$

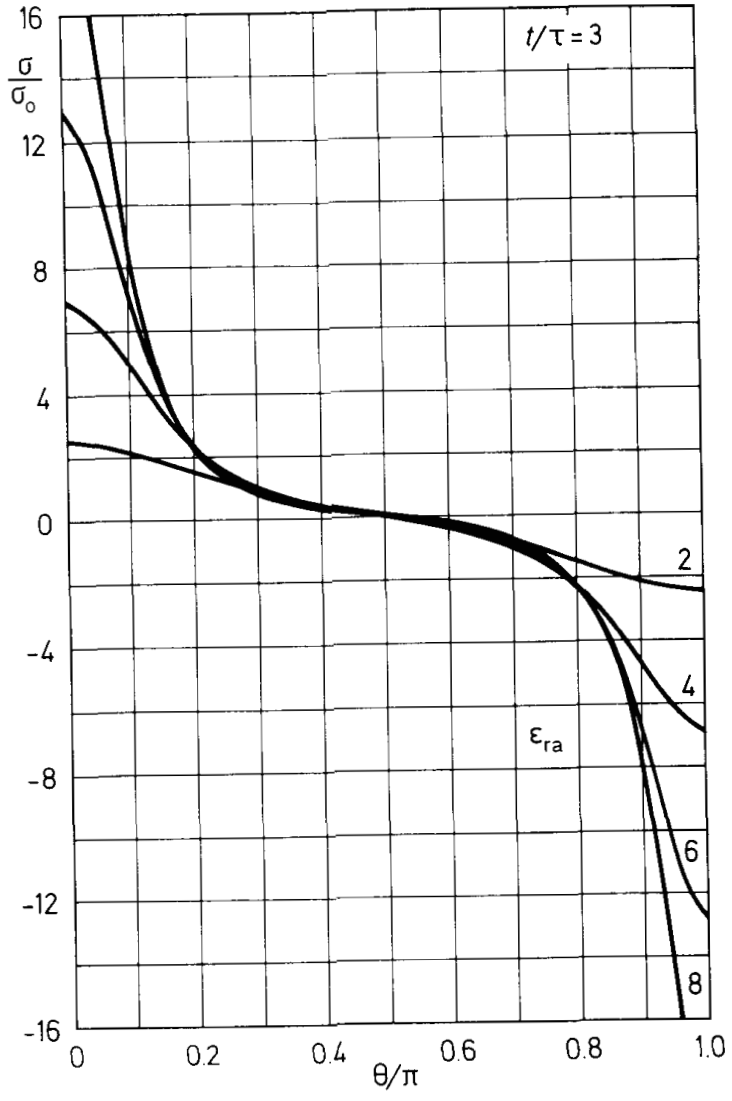

Figure 3.

Spatial variation of surface charge density $\sigma$ at void wall: infuence of bulk permittivity.

where

$$
E_{\mathrm{o}}=\frac{q}{4 \pi \varepsilon_{\mathrm{o}} R^{2}}
$$

It should be remembered that along the void axis $\theta=0$ or $\theta=\pi$, and hence $P_{2 n+1}(\cos \theta)= \pm 1$ for $0 \leqslant r / R \leqslant 1$

To illustrate the influence of the bulk polarization upon the $\vec{E}$ field in the void, the variation of $E_{a x}$ at time zero is shown in Figure 5 for different bulk permittivities. $\varepsilon_{r a}=1$ corresponds to the field between two point charges. From this diagram it is clear that the larger $\varepsilon_{r a}$ is the smaller is the field in the void.

The bulk permittivity has also an influence upon the rate at which the axial field decays. This aspect is indicated in Figure 6, which illustrates the variation of $E_{a x}$ for different values of $\varepsilon_{r a}$ at $t / r=3$. These field distributions are generated from the $\sigma$ distributions shown in Figure 3, from which the influence of $\varepsilon_{r a}$ upon $E_{a x}$ can be readily appreciated. 


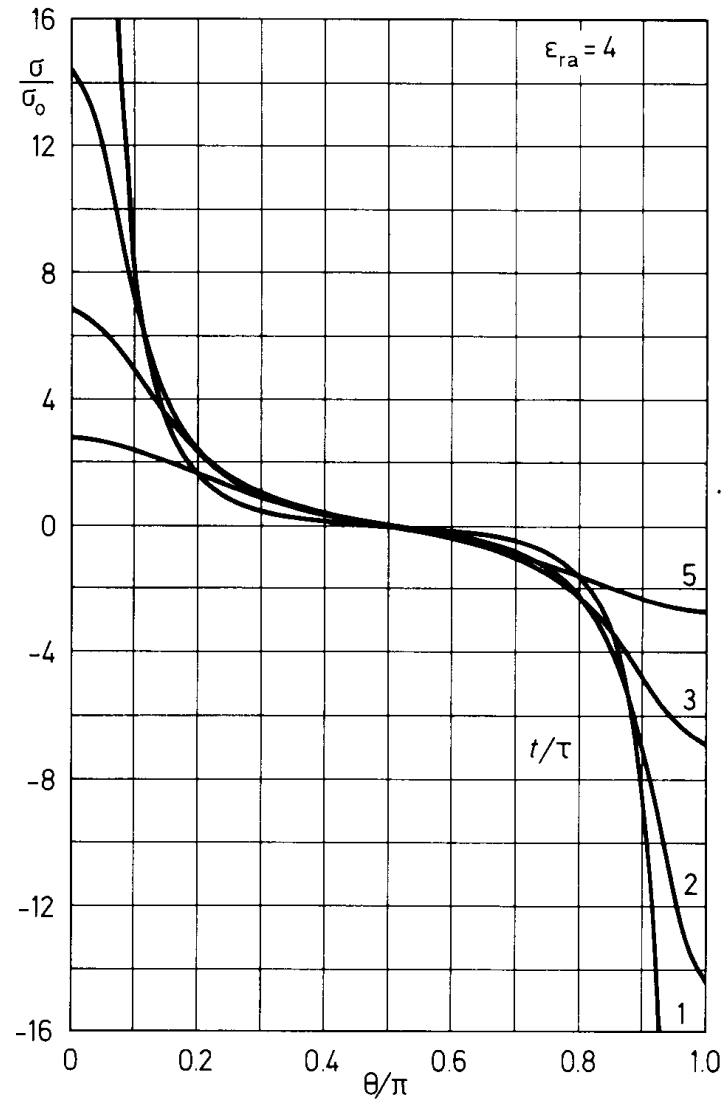

Figure 4.

Spatial variation of surface charge density $\sigma$ at void wall: influence of time.

The temporal development of $E_{a x}$ for $\varepsilon_{r a}=4$ is displayed in Figure 7 . The graphs indicate that as time increases, the void field decreases in magnitude and its degree of nonuniformity is steadily reduced. The corresponding $\sigma$ distributions are given in Figure 4.

\section{DISCUSSION AND CONCLUSION}

$\mathrm{O}^{\mathrm{n}}$ the basis of the temporal potential distribution asociated with a charged dielectric particle (cf. gas/ solid), it has been possible to derive the corresponding distribution for a wall charged void. This void potential distribution is derived by not only interchanging the media permittivities (cf. solid/gas), but also by taking account of the inherent zero net charge condition in a void. This zero net charge condition is fulfilled by superimposing a second particle type solution for a source

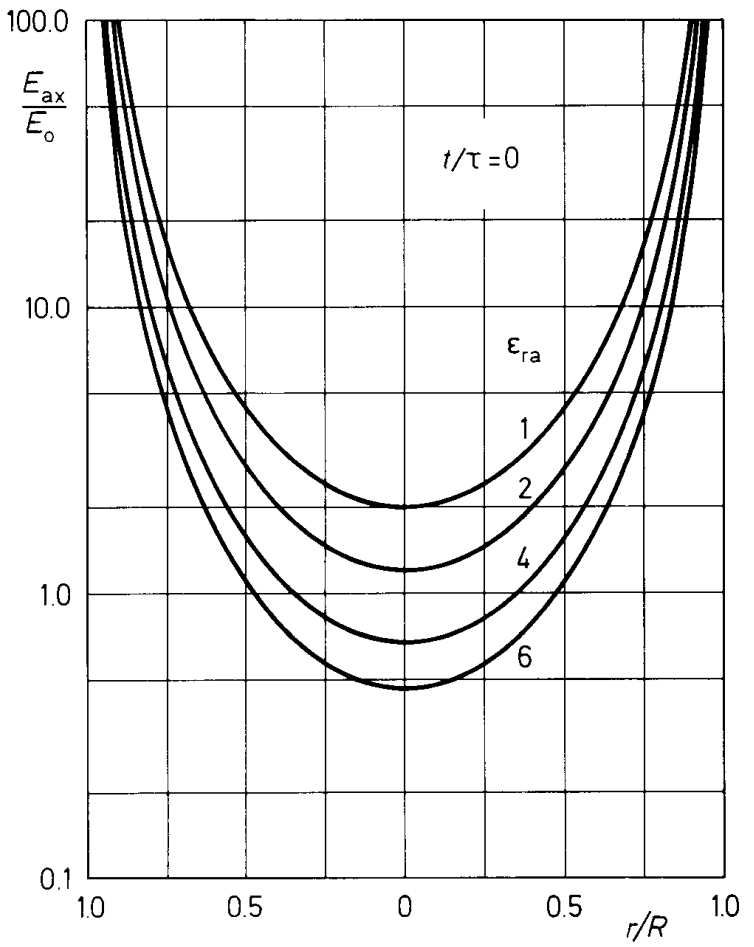

Figure 5.

Variation of the void axial electric field $E_{a x}$ at time zero: influence of bulk permittivity.

charge of opposite polarity. Although partial discharges in a gaseous void create charges, it should be remembered that, as the void is a closed volume, the net charge in the void remains zero.

The examination of the wall charge decay indicates that, in terms of the basic time constant $\varepsilon_{0} R / \Gamma$, this process can take a considerable time. The decay rate is significantly reduced by an increase in the permittivity of the bulk medium. The dominating influence of this permittivity is likewise reflected in the increased duration and thereby prolonged inhomogeneity of the electric field sustained in the void. However the absolute value of this field is reduced with an increase in bulk permittivity.

In conclusion, the present choice of a point charge to simulate the wall charge has the disadvantage that such a source is associated with a field singularity, and thus it is not possible to represent the maximum field at the void wall in a realistic manner. To overcome this limitation, other representations of wall charges are presently under examination. 


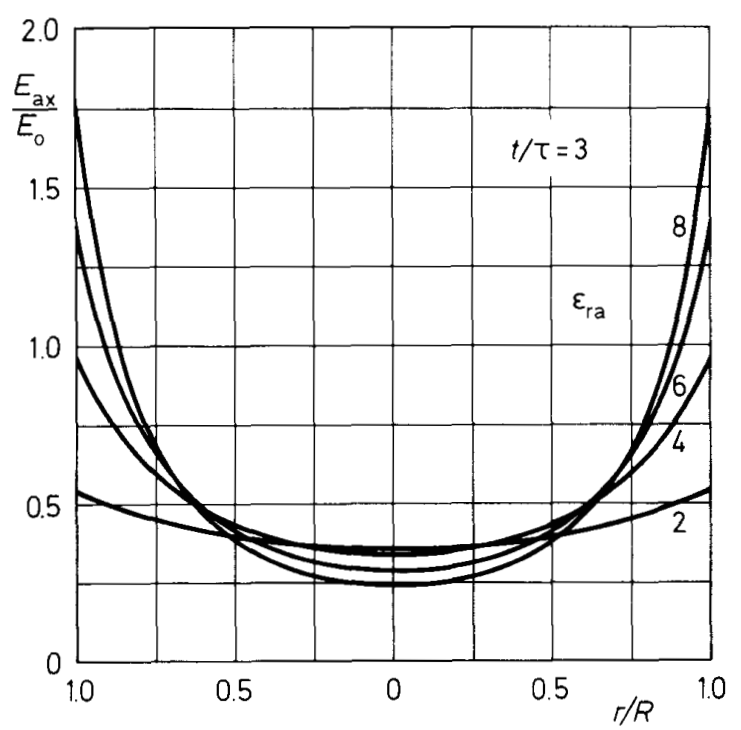

Figure 6.

Variation of the void axial electric field $E_{a x}$ : influence of bulk permittivity.

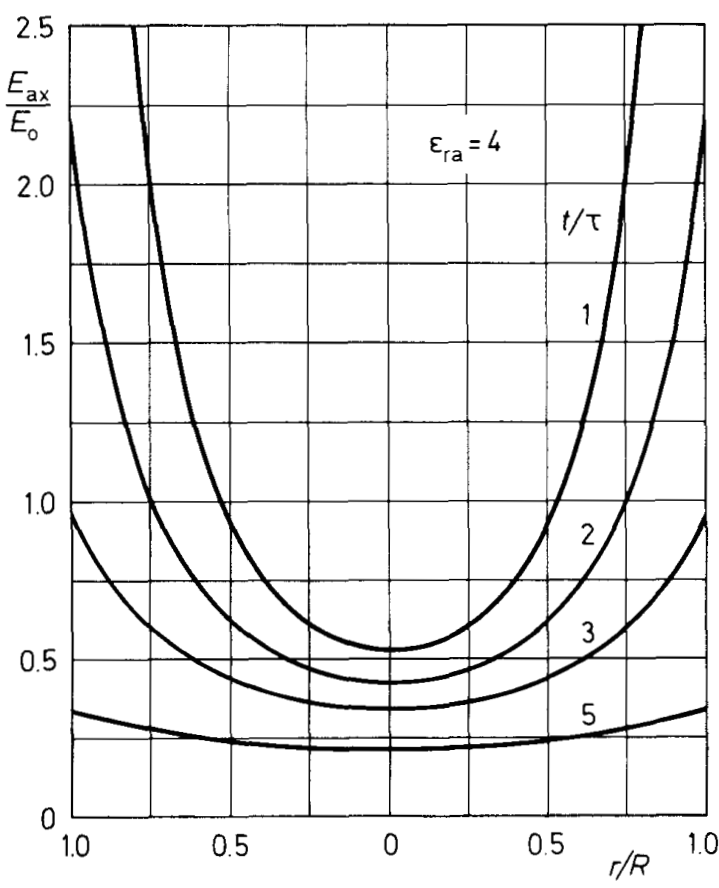

Figure 7.

Variation of the void axial electric field $E_{a x}$ : influence of time.

\section{REFERENCES}

[1] I. W. McAllister, "Surface Current Density $\vec{K}$ : An Introduction", IEEE Trans. Elect. Insul., Vol. 26, pp. 416-417, 1991.

[2] I. W. McAllister, "Electric Fields Associated with Transient Surface Currents", J. Appl. Phys., Vol. 71, pp. 3633-3635, 1992.

[3] J. A. Stratton, Electromagnetic Theory, McGrawHill New York 1941.

[4] E. Weber, Electromagnetic Fields, Mapping of Fields, Vol. I, J. Wiley New York 1950.

An initial version of this paper was presented at the 7 th International Symposium on High Voltage Engineering, Dresden, Germany, 1991.

Manuscript was received on 20 December 1991, in revised form 11 May 1992. 